\title{
Confocal Laser Scanning Microscopy
}

BioTechniques 27:992-1004 (November 1999)

\author{
Stephen W. Paddock \\ University of Wisconsin, \\ Madison, WI, USA
}

\begin{abstract}
Many technological advancements of the past decade have contributed to improvements in the photon efficiency of the confocal laser scanning microscope (CLSM). The resolution of images from the new generation of CLSMs is approaching that achieved by the microscope itself because of continued development in digital imaging methods, laser technology and the availability of brighter and more photostable fluorescent probes. Such advances have made possible novel experimental approaches for multiple label fluorescence, live cell imaging and multidimensional microscopy.
\end{abstract}

\section{INTRODUCTION}

Confocal microscopy came of age when a confocal laser scanning microscope (CLSM), useful for routine imaging of fluorescently labeled biological specimens, was introduced in the late 1980s (64). The instrument "enraptured the world's biological community with its exquisite and convincing illustrations of the power of the confocal laser scanning microscope" (28).

The popularity of the CLSM is evidenced by the increase in the number of confocal systems in use today. Their spectacular images continue to adorn scientific journals, textbooks and Web sites around the world. A seminal paper, published in 1987, demonstrated the universal application of a CLSM designed specifically for collecting images from relatively thick and fluorescently labeled biological specimens (65). The resolution of the images and their speed of collection finally alerted the biomedical community to the power of the confocal approach.

A major advantage of the CLSM was immediately clear to microscopists: its ability to capture in a digital image those details of fluorescently labeled tissue specimens that previously could be seen only by eye in a conventional epifluorescence microscope. Such images were technically challenging to capture on film because the background produced by fluorescence, either from labeled structures within the volume of the specimen or from naturally occurring autofluorescence, overwhelmed the signal from the focal plane of interest. Structures specifically labeled by immunofluorescence could be viewed by eye in the microscope field but were often impossible to document in a publication. Often, it would take days to produce an acceptable image on film. With the confocal approach, it takes only seconds to produce an acceptable digital image of an optical section of the specimen.

The term "optical section" refers to the instrument's ability to produce sharper images of fluorescently labeled tissues than those produced using a standard epifluorescence microscope and, in most cases, without resorting to physical sectioning of the tissue. The CLSM produces optical sections by scanning the specimen point-by-point with a laser beam focused in the specimen, and using a spatial filter (usually a pinhole or a slit) to remove unwanted fluorescence from above and below the focal plane of interest. Optical paths of the various commercially available CLSMs are designed so that when the laser beam is focused in the specimen, it is confocal with the point of light focused at the pinhole in front of the photodetector. Thus, only information from the focal plane of interest reaches the photodetector. The power of this approach lies in its ability to image structures at discrete levels within an intact biological specimen with a theoretical lateral resolution of $0.14 \mu \mathrm{m}$ and a vertical resolution of $0.23 \mu \mathrm{m}$ with a lens of 1.4 numerical aperture (NA) (67).

This article focuses on improvements made to the CLSM over the past decade and some of the experimental approach- 
es that resulted. Articles cited include details of the technical aspects of the range of confocal imaging systems, including disc scanning systems and related technologies such as deconvolution methods (7) used for producing optical sections $(47,56,67)$. Protocols for preparing specimens for use with these instruments are also cited $(34,46)$.

\section{EARLY CLSMs}

CLSMs in use today, while based on the principle of confocal imaging as outlined in Marvin Minsky's 1952 patent (35), are a result of improvements made to microscopes introduced in the 1980s. There are several different designs of CLSM, and they are usually classified by the way they scan specimens.

The earliest instruments, typified by Minsky's design, were stage-scanning systems that used a bright zircon light source rather than a laser. Here, the beam remained stationary, and the stage was moved in a precise raster pattern in order to scan the specimen. This design has the advantage of always scanning on the optical axis of the microscope and, consequently, avoids lens defects. Such laser based stage-scanning systems are especially useful for imaging larger specimens. Traditional$1 y$, these instruments have been used in the electronics industry in the manufacture and quality control of microcircuits. The systems are currently undergoing a renaissance in biomedical applications for screening arrays of fluorescently labeled DNA oligonucleotides on glass wafer supports or "gene chips" $(18,30)$.

In contrast to these early instruments, CLSMs introduced in the late 1980s scanned a stationary specimen with a laser beam. With the availability of affordable lasers and improved imaging systems, these beam-scanning instruments became more practical to produce commercially. The beam-scanning approach has proven to be more suitable for most biological applications than the stage-scanning systems because the image acquisition times are faster than the stage-scanning systems. Typical scanning speeds are from 1 frame/s to near video rates or 30 frames/s, while the stage-scanning sys- tem's speed is 1 frame/10 s. Also, using the beam-scanning instruments means less chance of vibration artifacts caused by movement of the specimen.

The early CLSMs were designed to interface with a conventional epifluorescence microscope, rather like a video camera. The laser was attached to a scan head mounted above an upright or below an inverted light microscope stand. Scanning the laser beam in the $\mathrm{X}$ and the $\mathrm{Y}$ direction across the specimen was achieved using two mirrors that were usually driven by galvanometers and controlled by a computer. Movement in the $\mathrm{Z}$ direction was carried out using a stepper motor attached to the fine focus of the microscope. The computer also controlled the position and fine movement of the stepper motor. Zooming the image was achieved by changing the area of the specimen scanned by the mirrors.

The signal from the specimen was detected by a photomultiplier tube (PMT) positioned just behind a pinhole. The PMT detected only light focused at the pinhole; light from above and below the plane of interest in the specimen was prevented by the pinhole from striking the PMT. The output from the PMT was built up in a digital framestore in a microcomputer; it was displayed as an image on a video monitor screen or stored as a digital file. Images were collected at approximately one frame per second, and signal averaging was usually necessary to eliminate much of the background noise (usually from the PMT) in the images. Many systems were equipped with a second PMT that enabled two images to be collected simultaneously at different excitation wavelengths.

In these early CLSMs, the amount of light reaching the specimen was controlled by attenuating the output from the laser using a range of neutral density filters mounted in a filter wheel placed by hand in the beam path between the laser and the scan head. The fluorescence filter sets for selecting the excitation wavelength from the laser and filtering the emitted wavelength from the specimen to the PMT were identical to those used for conventional wide-field epifluorescence microscopy. These filters were mounted in filter blocks that the user slipped by hand in and out of the scan head. Minor alignments of the beam path were required after each filter change. The diameters of the pinhole and the gain and black level of the PMTs were adjusted by hand.

\section{TECHNICAL IMPROVEMENTS IN THE 1990s}

Today's CLSMs are relatively sophisticated and versatile when compared with earlier models. All parts of the imaging chain (Figure 1) have been improved. Now included are stable multiwavelength lasers; optical filters with improved specifications and polychroic mirrors; more efficient mirrors (as much as $99 \%$ reflecting); and high NA water immersion lenses that have long working distances and efficient detectors, such as low noise PMTs or charge-coupled devices (CCDs). Today's affordable computers have larger, faster hard disks with more random access memory (RAM), improved software and, above all, improved facilities for the display, reproduction and archiving of images.

In most of the CLSMs of the 1990s, the laser has been moved away from the scan head and its output is transferred by a fiber optic cable. This design reduces the transmission of vibrations from the cooling fan of the laser to the microscope itself. Such vibrations appear as irregular horizontal lines in the images and reduce the resolution in the final image. The fiber optic cable launch also facilitates addition of extra lasers to the system. It simplifies alignment and maintenance by making the lasers more accessible. The systems are easier to transfer between upright and inverted microscope stands; some are now mounted directly on the optical axis of inverted microscopes.

The trend has been to make CLSMs more user friendly. Many operations now are automated, controlled directly from the computer keyboard rather than by hand as before. Laser, brightness, for example, is controlled by computer using a range of neutral density filters that are mounted in a motorized filter wheel placed in the beam path between the laser and the scan head or by direct control of laser power. Many optical filters are mounted on a second computer-controlled filter wheel in the scan head. 
Likewise, computers control the pinhole diameter, and the gain and the black level of the PMTs. Thus, the instrument is more easily configured using software control so that a novice can collect acceptable images in less time-a useful feature for busy multi-user facilities.

\section{IMAGING MODES}

The main application of the CLSM is still to collect single optical sections from fixed and immunofluorescently labeled specimens in single, double, triple and multiple wavelength modes. The CLSM also collects Z-series, or series of optical sections, which can be combined as optical projections, displayed as montages or processed further into stereo pairs or 3-D reconstructions (46). Single $\mathrm{X}-\mathrm{Z}$ sections can also be collected by scanning a line at different Z-depths.

To image living cells, single optical sections are usually collected at preset time intervals (time-lapse imaging) or Z-series are collected over time (4-D imaging). The 4-D approach is especially useful for following the movement of structures in the $\mathrm{X}, \mathrm{Y}$ and $\mathrm{Z}$ planes.

Additional microscopic modes of collecting images with the CLSM include a nonfluorescent, reflected light mode. Here, backscattered light, either from naturally reflective material in the specimen or from gold or silver particles, is collected as a signal (41).

CLSMs also collect simultaneously transmitted light images and confocal fluorescence images by using a detector that collects any light from the same scanning beam passing through the specimen. All basic modes of light microscope imaging can be collected using the transmitted light detector. They include bright field, differential interference contrast, phase contrast and dark field, but these images are not improvements over images produced by a conventional transmitted light microscope. Because these images are collected with the same laser beam as the confocal images, they are in perfect register with one another. This feature is extremely useful for mapping the confocal fluorescence image, which often appears rather abstract, onto familiar landmarks in the specimen that can only be imaged using the transmitted light mode $(9,54)$.
A real-color transmitted light detector is now available on those CLSMs equipped with three PMTs. The device collects white light (usually from a krypton argon laser) that has passed through the specimen through red, green and blue (RGB) filters simultaneously into the RGB channels. The images are then merged into a single RGB image to produce a real-color image similar to that from some color digital video cameras (29).

\section{MULTIPLE LABELS IN THE 1980s}

CLSMs in the 1980s collected two grayscale images simultaneously from double-labeled specimens by using the $514 \mathrm{~nm}$ line of a single argon laser to excite both fluorescein (suboptimally) and rhodamine dyes. The emitted light from the labeled specimens was then filtered to two photomultipliers using a dichromatic mirror. Unless the user took great care preparing the specimen to balance the brightness of the signal emitted from the two fluorochromes, part of the fluorescein signal often appeared in the rhodamine image as bleedthrough. This signal could be removed from the image by digitally subtracting that part of the image caused by bleedthrough from the other channel.

Image artifacts caused by one channel bleeding into another could be avoided by imaging the two fluorochromes sequentially using two different filter sets that were better matched to the optimum excitation of the fluorochromes. However, this method could lead to misregistration of the images caused by movement of the specimen during imaging, slight differences in the angles of the dichromatic mirrors in the filter blocks, slight differences in positioning the filter blocks in the scan head or by using an objective lens that was poorly corrected for chromatic aberration so that different wavelengths of light were focused at different levels in the specimen. Artifacts caused by misalignment of the images could be rectified using digital methods and were usually more noticeable in higher magnification images.

Occasional problems arose with photobleaching of the fluorochromes using sequential imaging. For example, when collecting the first image, the second fluorochrome was bleached to such an extent that it was impossible to collect an image from the same region of the specimen.

Several years ago, an improved aircooled krypton argon ion laser was added to the CLSM as a convenient source for multiple label applications (5). The laser delivers three major lines at $488 \mathrm{~nm}, 568 \mathrm{~nm}$ and $647 \mathrm{~nm}$, which are conveniently matched to the excitation spectra of three of the most commonly used classes of fluorophores: fluorescein, rhodamine and cyanine 5, at the time a new dye $(6,13)$.

The first strategy for collecting images from triple-labeled specimens was to collect the three images sequentially at the three different excitation wavelengths by changing the filter blocks by hand, collecting the three images into separate computer files, and then colorizing and merging them, usually with a separate computer equipped with a 24bit color card $(5,42,45)$. This method was introduced because, at the time, confocal host computers typically had an 8-bit card and could only combine two images by reducing their pixel resolution into a single merged 8-bit image. This approach to imaging multiple labeled specimens, while successful and less prone to bleedthrough problems, was labor intensive and slow.

\section{MULTIPLE LABELS IN THE 1990s}

Today, CLSMs are available that collect three images simultaneously from triple-labeled specimens. No information from the fluorophore is lost because the emission from all three images is detected at the same time. The light path in these instruments has been designed to filter the emitted signal from the three fluorophores to three separate PMTs (Figure 1). Each PMT has a pinhole situated in front of it that can be adjusted independently. The computer has enough power to combine and display the three 8-bit images during scanning, with no loss in resolution (5). The simultaneous method is much faster than collecting the images sequentially, and some adjustment for 
bleedthrough between channels can be made during the collection of images rather than after imaging.

The collection of multiple label images is achieved using a system equipped with a single krypton argon laser. Or three separate lasers can be used in combination with the fiber optic launch, for example, the $488 \mathrm{~nm}$ line of an argon laser for the fluorescein channel, a green helium neon laser (543 $\mathrm{nm})$ for the rhodamine channel and a red diode laser $(635 \mathrm{~nm})$ for the cyanine 5 channel. The three-laser config- uration is becoming more popular as the cost of individual lasers decreases and their lifetimes increase in relation to the lifetime of a single air-cooled krypton argon laser.

More recently available CLSMs have an acousto-optical tunable filter (AOTF) incorporated into the light path. The AOTF provides fine control of the intensity and the wavelength of light passing through it (26). Using the AOTF, each excitation wavelength is selected and its brightness is controlled with microsecond time resolution, which therefore replaces both neutral density and excitation filters in the excitation pathway (Figure 1). Moreover, using the AOTF, the excitation wavelengths can be more closely matched to the excitation maxima of the fluorochromes used in the specimen. This helps to reduce background autofluorescence from the specimen and reduces bleedthrough from one channel to another. The AOTF can be adjusted to compensate for variations in laser output, a feature that is especially useful as the laser tube begins to age and its out-

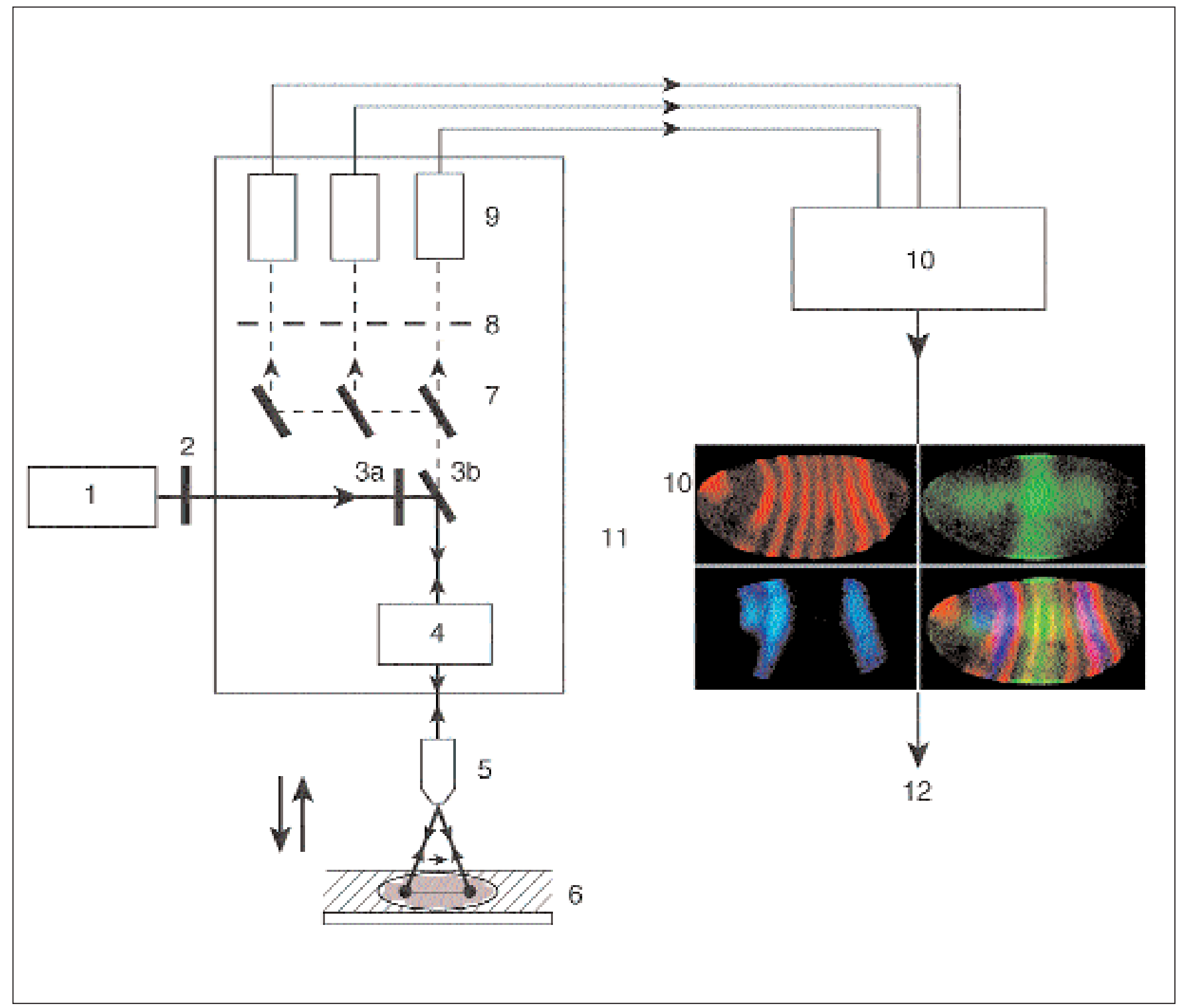

Figure 1. Information flow in a generic CLSM of the 1990s. One or more lasers (1); neutral density filters mounted in a filter wheel (2); excitor (3a) and dichromatic filters (3b); scanning unit (4); objective lens (5); specimen (6); emission filters and dichromatic mirrors (7); pinholes (8) placed in front of one or more PMTs (9); computer and digital control unit (10); high resolution video display (11); digital images are exported to high resolution digital printers, to other computers or to the World Wide Web (12). 
put in various channels begins to decrease. For example, when an air-cooled krypton argon tube begins to age, the cyanine 5 line usually weakens in intensity relative to the two other lines.

\section{MULTIPLE LABEL APPLICATIONS}

Strategies for detecting three fluorescent probes simultaneously are used for mapping the relative distribution of three proteins within cells and tissues (Figure 2). The protocols use three fluorescently labeled antibody probes or combinations of antibody and direct labels. In this way, specific proteins can be mapped to cellular compartments using label combinations of known landmarks in the cell, using, for example, rhodamine phalloidin to label cell outlines (11), ToPro ${ }^{\mathrm{TM}}$ (Molecular Probes, Eugene, OR, USA) to label the nuclei and an antibody of unknown distribution on the third channel. Indeed, a wide range of fluorescent probes are available for labeling specific cellular compartments (24).

A popular application of multiple labeling is to map gene expression in genetically modified Drosophila tissues. Here, clones of cells can be produced that either express a gene in an ectopic position or remove gene expression from its wild-type position in a field of cells (3). Multiple label confocal microscopy is used as an assay to detect the positions of the clones and their effects on gene expression patterns and tissue morphology. These clones of cells are usually visualized using a myc-epitope tag on one fluorescent channel, and proteins of interest and/or direct labels are placed on the other fluorescent channels $(2,27,38)$.

While immunofluorescence continues to be a major technique for mapping the distribution of specific macromolecules within cells and tissues using the CLSM, fluorescence in situ hybridization (FISH) has become a powerful and complementary technique. DNA and RNA sequences are localized in cells using bright nonradioactive fluorescent probes that can be detected with modern CLSMs (1). Proteins and transcripts can be mapped in the same specimen using multiple labeling protocols that combine immunofluorescence and FISH $(22,53,62)$ and the CLSM for imaging. Triple label strategies have also been developed for imaging apoptosis, FISH and nuclear staining using ToPro (14).

Over 20 probes have been labeled by FISH and imaged on chromosome spreads using combinatorial labeling strategies. To date, however, conventional fluorescence microscopy using a CCD camera has been used for collecting the images $(55,57)$. The method may be an option for labeling thicker tissues, using the CLSM to collect the images and the existing software to combine and display the images.

\section{LIVING CELLS}

The early confocal imaging systems, while excellent for imaging fixed and fluorescently labeled specimens, were not exceptionally well designed for imaging living cells because their light budgets were rather inefficient. For example, just $0.2 \%$ of the overall fluorescence leaving the specimen actually reached the photodetector in an early CLSM; only $0.02 \%$ of it reached the photodetector if the pinhole was closed (52). In many cases, this meant that the cells were killed by the amount of light required to produce an acceptable image and the pinhole was usually set to a suboptimal (open) position to collect the valuable photons from the specimen. A compromise between image quality (size, number of scans, etc.) and photodamage to the cells had to be made.

Nevertheless, when care was taken to protect the cells from long exposures to damaging laser light, and antioxidants such as ascorbic acid were included in the medium surrounding the cells, studies of living cells and tissues were successfully completed using the early instruments. For example, the dynamics of the Golgi apparatus were visualized in astrocytes labeled with NBD-ceramide using an early CLSM (12).

As improvements were made to the photon efficiency of various CLSMs, the success rate of live cell experiments has increased and more and more tissue types have been successfully imaged
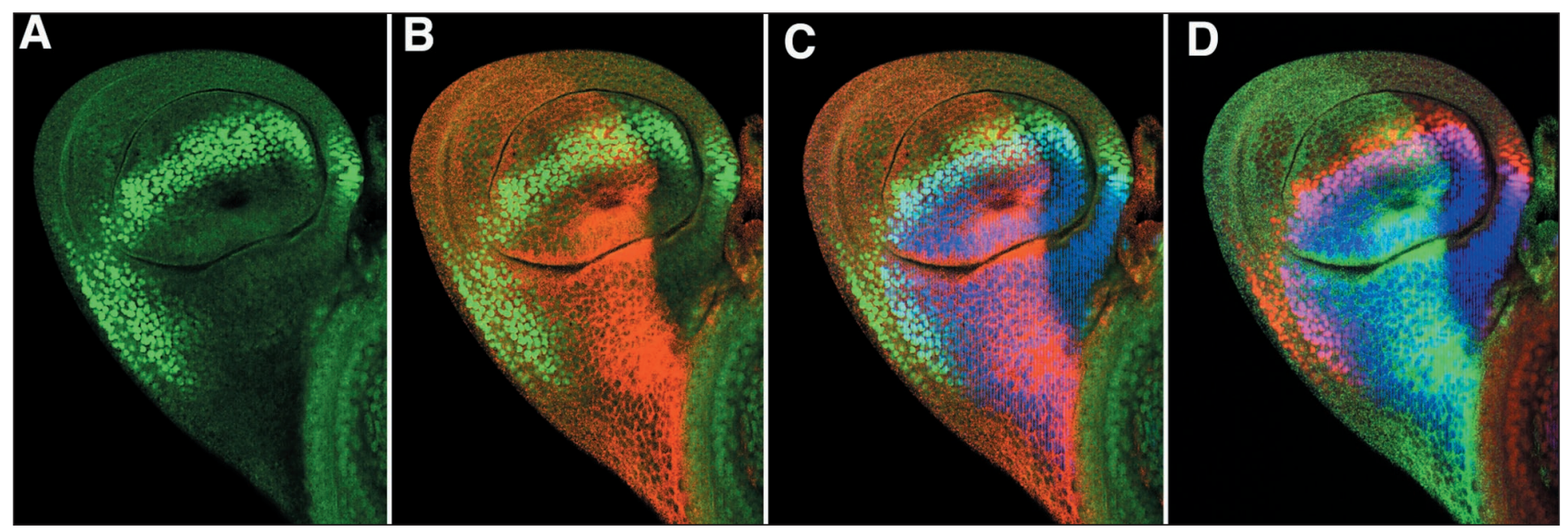

Figure 2. Multiple labeling of a Drosophila third instar haltere imaginal disc. Single label vestigial expression in green (A); double label vestigial and CiD in red (B); triple label vestigial, $\mathrm{CiD}$ and apterous in blue (C); and with the grayscale images assigned to different colors in (D) by rearranging them in PhotoShop using methods previously described $(42,44)$. 
(59). These improvements include the addition of PMTs with higher quantum efficiency; more efficiently reflecting mirrors; lenses with improved light transmission including high NA water immersion lenses; and faster computer hardware and software written specifically for the collection of data sets from living cells. Such additions have improved both the temporal and the spatial aspects of time-lapse imaging using the CLSM.

Brighter and less phototoxic dyes have been developed for live cell imaging. This is epitomized by the green fluorescent protein (GFP), which is currently an extremely useful probe for imaging gene expression in living tissues (58). GFP is a relatively small (27
$\mathrm{kDa}$ ) and naturally fluorescent molecule that is resistant to photobleaching. Because one of the most common forms of GFP fluoresces in the blue range, it can be imaged with little or no modification to the CLSM using a standard fluorescein filter set or a modified set that reduces background autofluorescence from unlabeled tissues or reagents (40). GFP has now been used in many studies of living cells with the CLSM (21). Spectral variants of GFP are available for live cell imaging of multiple labeled specimens $(25,51)$ and fluorescence lifetime microscopy (48). A method for labeling recombinant proteins in living cells has been reported recently (23).

The introduction of CLSMs with

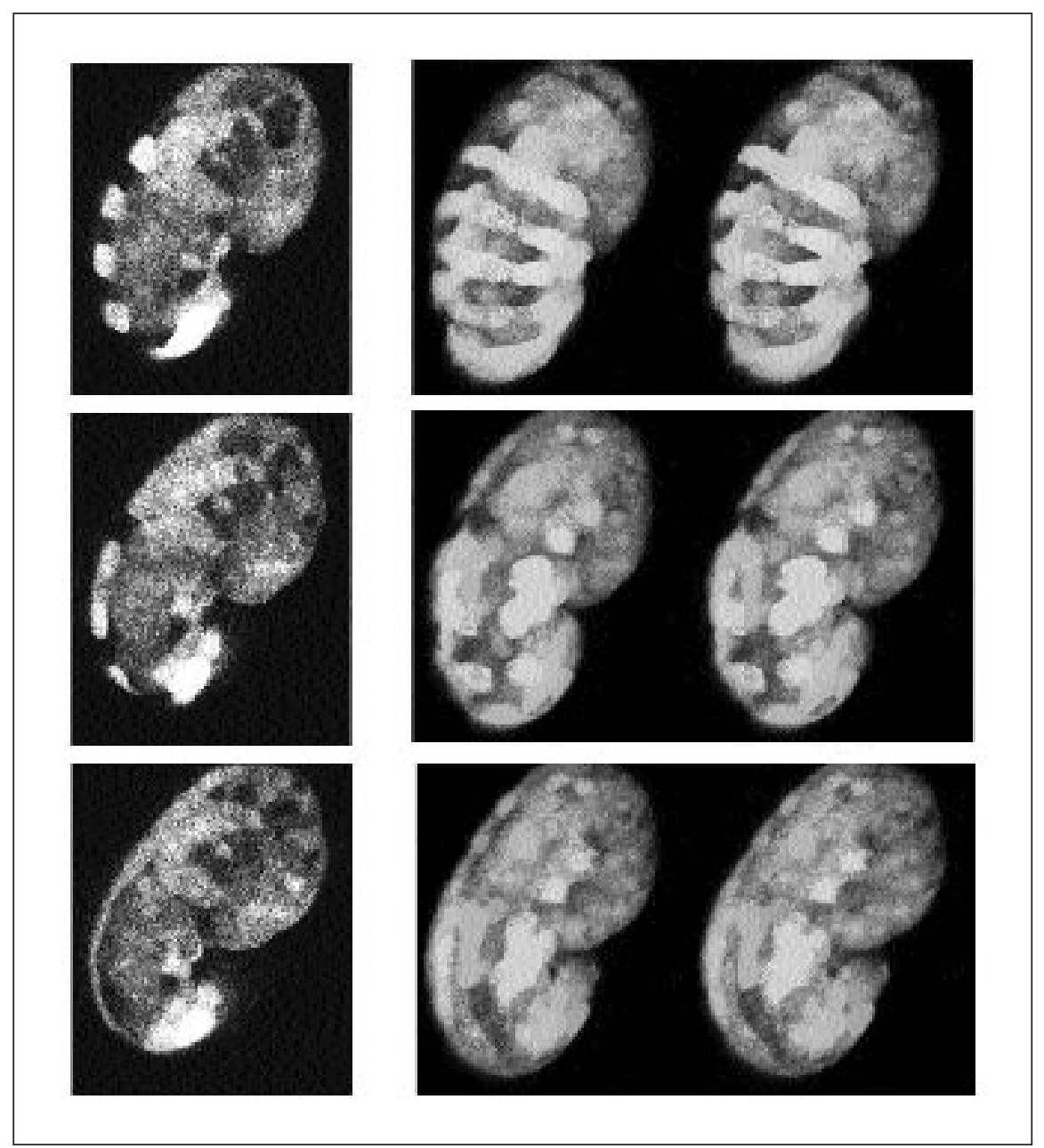

Figure 3. Four-dimensional recording of a developing C. elegans embryo. Cytoplasmic GFP expression within alternating dorsal hypodermal cells was imaged before (top), during (middle) and after (bottom) fusion of these cells with their non-expressing neighbors. Images were recorded by two-photon laser scanning microscopy using a $900 \mathrm{~nm}$ Ti:Sapphire excitation laser. Left column shows a single optical section followed over time. Right column shows stereo pairs representing full 3-D reconstructions of each time point. 
improved photon efficiency has enabled the imaging of fluorescently labeled proteins injected into cells. Historically, a problem with using the CLSM for imaging such specimens was that fluorochromes bound to each protein molecule were photobleached too quickly to allow for the collection of long movie sequences. Now, improvements to the photon efficiency of modern CLSMs allow lower light levels for imaging so that the fluorochromes are bleached more slowly and longer time-lapse sequences can be collected. For example, the dynamics of rhodamine-labeled tubulin have been visualized in Drosophila embryos for the analysis of defects during abnormal development of mutants (15). These studies have additional value because they confirm the existence of structures that have only been visualized in fixed and stained tissues.

Many fluorescent probes are now available for imaging the dynamics of various ions in cells (61). Perhaps the most prevalent use of fluorescent probes with the CLSM in recent years has been for imaging calcium dynamics. To control for various experimental artifacts (e.g., photobleaching of the fluorophore), it is necessary to use ratio imaging. A common method is to ratio Fluo3 and FURA-red ${ }^{\mathrm{TM}}$ (Molecular Probes) (33), two probes that are excited by the lasers on most commercially available CLSMs, where software designed for physiological imaging is usually available. The confocal systems that are equipped with multiple photodetectors are excellent for such studies.

For imaging fast biological events (19), video-rate CLSMs were introduced. For example, waves of calcium have been imaged in retinal glial cells labeled with calcium green where each frame was collected as an average of 16 video frames every $0.93 \mathrm{~s}$ (39). The point scanning systems can be used in a line scan mode for measuring fast events where each line is collected at millisecond time resolution, although the scan rate is clearly too slow for collecting images at full spatial resolution. Calcium sparks in heart muscle cells have been visualized using the calcium indicator Fluo-3, where each line scan was acquired at a rate of $2 \mathrm{~ms}(8)$, for example.

\section{MULTIPLE PHOTON IMAGING}

Multiple photon imaging has further improved the chances of successfully imaging living cells $(17,50,66)$. Using the two-photon approach, only those fluorophores in the focal plane being imaged are excited; cells are less susceptible to photodamage by the longer wavelength laser light (unless they contain pigments that absorb the red light). In a recent study of living nematode embryos, a total of 2160 optical sections was collected from a single living embryo using two-photon imaging with no apparent photodamage to it $(36,37)$. This approach has enabled the collection of Z-series over a considerable time period with little or no photodamage to the specimen or photobleaching of the probes (Figure 3).

Multiple photon imaging uses the same scanning method as the CLSM but dispenses with the pinhole to prevent out-of-focus fluorescence from reaching the photodetector. Instead, the twophoton microscope relies on the phenomenon of multiple photon excitation of fluorophores. This phenomenon occurs only above a certain energy threshold and is confined to the focus of the laser in the specimen in which the energy levels are high enough. That is, when a high energy beam is focused in the specimen, only fluorochrome molecules in the plane of focus are excited by two or three photons of light interacting with a fluorophore molecule simultaneously. At the moment, the approach uses expensive high-energy pulsed lasers for excitation of the fluorochromes, but the technique is becoming more prevalent with the introduction of more affordable solid-state lasers.

The multiple photon approach has several advantages when compared to the CLSM for imaging living cells. Optical sections can be collected from deeper inside the specimen than those collected with the CLSM. Also, the multiple photon approach yields no fluorescence above or below the plane of focus to attenuate the light leaving the specimen. In addition, there is less light scattering from the longer wavelength beam of the laser, which aids in deeper penetration of the beam into the specimen than with the CLSM approach $(49,50)$.

Moreover, UV fluorophores [e.g., Hoechst 33342 and cellular components that could previously only be imaged using a specialized UV CLSM with UV optics and a UV laser (4)] can now be imaged using conventional optics and multiple photon excitation $(68,69)$.

Multiple labeled specimens can also be imaged using the multiple photon approach (69). For example, rhodamine can be excited by two photons of red light in the same specimen simultaneously, while DAPI can be excited by three photons of red light. In this way, a range of fluorochromes can be imaged in a single specimen by filtering the light emitted from the specimen to different PMTs or by sequentially changing the emission filter to a single PMT. Four-color imaging of rat basophilic leukemia cells was achieved using a single $700 \mathrm{~nm}$ laser to excite four different fluorochromes from which emission was collected into four different channels (69). 


\section{MULTIDIMENSIONAL IMAGING}

Multidimensional imaging refers to the practice of collecting images at different depths and/or time points. It is an increasingly practical option with the development of more sensitive CLSMs and the introduction of practical multiple photon imaging. Additionally, specimens may be labeled with multiple probes, which adds the dimension of wavelength. Yet more fluorophores can be imaged in a single multiple-labeled specimen using a confocal system that combines multiple photon excitation and conventional one photon excitation. Soon, the number of fluorochromes imaged using the confocal approach will depend on the number of lasers attached to the imaging system.

The resolution of the CLSM is a little better than that of the conventional epifluorescence microscope but not as good as that achieved with an electron microscope. Additional information can be collected from a single specimen using more than one type of microscope, for example, by combining the confocal approach with electron microscopy to image the same region of a specimen (16).

An important feature of multidimensional images collected with the CLSM is that they are usually in register with one another. This feature includes optical or Z-series and images collected at different wavelengths from the same region of the specimen, either sequentially or simultaneously, including the transmitted light images. This facilitates the display of multidimensional images using computer reconstruction of Z-series and the combination of multi-wavelength images.

Living cells are commonly imaged using time-lapse methods. These files are usually displayed as a video sequence directly from the CLSM to a video recorder or optical memory disc recorder (OMDR). A digital-to-analog video converter may be required to record digital images from the CLSM in real time onto video tape. Digital video methods have improved over the years so that most time-lapse sequences are displayed in digital format (63).

Early attempts at 4-D imaging were made by collecting Z-series at user- determined time intervals, and subsequently rendering a 3-D reconstruction at each time point and displaying the reconstructions in sequence as a digital movie (44). More recent software can collect sequential Z-series using the CLSM, and additional software can analyze and display such 4-D data sets (Figure 3). For example, programs are now available that display 4-D data sets as stereo movies or track the movement of a structure over time through consecutive stacks of images $(36,60)$ or measure changes in volume over time (20).

\section{PRODUCTION OF HARD COPIES}

Hard copies of the images from the early CLSMs were usually produced using a $35 \mathrm{~mm}$ camera mounted on a tripod to photograph the screen of the video monitor or with an analog video printer. Facilities for hard copy have improved dramatically over the past ten years, especially for color images, with the introduction of affordable high-resolution digital printers and slide makers $(31,32)$ and the improved ability to produce and show digital movies $(44,63)$.

An ever-increasing number of journals now accept images as single frames or as movies for publication in digital format. In addition to traditional print-based publications, they also publish digital versions that are available either as a subscription available over the World Wide Web or on compact discs mailed to the subscriber. Subscribers can even select an article from the journal and print a hard copy of it in their laboratories. Theoretically, digital publications should also save paper and avoid the problem of storing so many printed journals. The development of reusable "digital paper" is an attempt to dispense with traditional paper (10). By these means, images should be accessible to any computer for viewing at the same resolution and color balance as those collected directly from the host computer of the confocal microscope, depending on the final resolution of the output device in the laboratory (43).

\section{CONCLUSION}

Introduced in the late 1980s, the CLSM has been used routinely for imaging a wide range of fluorescently 
labeled biological specimens (46). Over the years, major improvements have been made at all stages of the imaging chain, from the reagents available for specimen preparation through the technological aspects of the microscopes themselves to the quality of the final images. While the vast majority of the CLSM's applications continue to be for imaging fixed and fluorescently labeled specimens in single and multiple wavelength modes, improvements made to the instruments over the past decade have made the imaging of living specimens in situ applicable to a much wider range of specimens.

New technology from a wide range of sources will continue to drive future developments of the instruments. Members of the biological community will continue to transfer new technology to the CLSM, and the vision and response of vendors who incorporate these innovations into a commercial product is vital. The introduction of multiple photon imaging by the addition of pulsed lasers to confocal scanning units is an exam- ple of such a process. However, the current trend is towards more complex and expensive instrumentation, which is usually beyond the budget of most individual laboratories.

Further developments should keep in mind the relatively simple needs of many users for a basic, low maintenance instrument for the routine collection of optical sections and one that falls within the budget of individual investigators. Ideally, such systems would be built in a modular design so that improvements and additions could be made without purchasing an entirely new system each time an innovation is introduced or a new research direction is contemplated.

\section{ACKNOWLEDGMENTS}

I would like to thank Leanne Olds for her expertise with drawing Figure 1; Jim Williams for providing the specimen for Figure 2; Bill Mohler for providing Figure 3; and last but certainly not least, Sean Carroll for our continued collaboration.

\section{REFERENCES}

1.Albertson, D.G., R.M. Fishpool and P.S. Birchall. 1995. Fluorescence in situ hybridization for the detection of DNA and RNA. Meth. Cell Biol. 48:339-364

2.Basler, K. and G. Struhl. 1994. Compartment boundaries and the control of Drosophila limb pattern by hedgehog protein. Nature 368:208214

3.Blair, S.S. 1995. Compartments and appendage development in Drosophila. BioEssays 17:299-309.

4.Bliton, C., J. Lechleiter and D.E. Clapham. 1993. Optical modifications enabling simultaneous confocal imaging with dyes excited by ultra-violet and visible-wavelength light. J. Microsc. 169:15-26.

5.Brelje, T.C., M.W. Wessendorf and R.L. Sorenson. 1993. Multicolor laser scanning confocal immunofluorescence microscopy: practical applications and limitations. Meth. Cell Biol. 38:98-177.

6.Brismar, H., O. Trepte and B. Ulfhake. 1995. Spectra and fluorescence lifetimes of lissamine rhodamine, tetramethylrhodamine isothiocyanate, texas red, and cyanine 3.18 fluorophores - influences of some environmental factors recorded with a confocal laser scanning microscope. J. Histochem. Cytochem. 43:699-707.

7.Chen, H., D.D. Hughes, T.A. Chan, J.W. Sedat and D.A. Agard. 1996. IVE (Image Visualization Environment): a software platform for all three- dimensional microscopy applica- tions. J. Struct. Biol. 116:56-60.

8.Cheng, H., W.J. Lederer and M.B. Cannell. 1993. Calcium sparks: elementary events underlying excitation-contraction coupling in heart muscle. Science 262:740-744.

9.Cohen-Cory, S. and S.E. Fraser. 1995. Effects of brain-derived neurotrophic factor on optic axon branching and remodeling in vivo. Nature 378:192-196.

10.Comiskey, B., J.D. Albert, H. Yoshizawa and J. Jacobson. 1998. An electrophoretic ink for all-printed reflective electronic displays. Nature 394:253-255.

11.Condic, M.L., D. Fristrom and J.W. Fristrom. 1991. Apical cell shape changes during Drosophila imaginal leg disc elongation: a novel morphogenetic mechanism. Development 111:23-33.

12.Cooper, M.S., A.H. Cornell-Bell, A. Chernjavsky, J.W. Dani and S.J. Smith. 1990. Tubulovesicular processes emerge from transGolgi cisternae, extend along microtubules, and interlink adjacent trans-Golgi elements into a reticulum. Cell 61:135-145.

13.Cullander, C. 1994. Imaging in the far-red with electronic light microscopy: requirements and limitations. J. Microsc. 176:281286.

14.Davis, W.P., Y.M.W. Janssen, B.T. Mossman and D.J. Taatjes. 1997. Simultaneous triple fluorescence detection of mRNA localization, nuclear DNA, and apoptosis in cultured cells using confocal scanning laser microscopy. Histochem. Cell Biol. 108:307-311.

15.Debec, A., R.F. Kalpin, D.R. Daily, P.D. McCallum, W.F. Rothwell and W. Sullivan. 1996. Live analysis of free centrosome behavior in normal and aphidicolin-treated Drosophila embryos. J. Cell Biol. 134:103115.

16.Deerinck, T.J., M.E. Martone, V. Lev-Ram, D.P.L. Green, R.Y. Tsien, D.L. Spector, S. Huang and M.H. Ellisman. 1994. Fluorescence photooxidation with eosin: a method for high resolution immunolocalisation and in situ hybridization detection for light and electron microscopy. J. Cell Biol. 126:901-910.

17.Denk, W., J.H. Strickler and W.W. Webb. 1990. Two-photon laser scanning fluorescence microscopy. Science 248:73-76.

18.DeRisi, J., L. Penland, P.O. Brown, M.L. Bittner, P.S. Meltzer, M. Ray, Y. Chen, Y.A. Su and J.M. Trent. 1996. Use of a cDNA microarray to analyze gene expression patterns in human cancer. Nature Genetics $14: 457-460$.

19.Draaijer, A. and P.M. Houpt. 1988. A standard video-rate confocal laser-scanning reflection and fluorescence microscope. Scanning 10:139-145.

20.Errington, R.J., M.D. Fricker, J.L. Wood, A.C. Hall and N.S. White. 1997. Four-dimensional imaging of living chondrocytes in cartilage using confocal microscopy: a pragmatic approach. Am. J. Physiol. 272:C1040C1051.

21.Endow, S.A. and D.J. Komma. 1996. Centrosome and spindle function of the Drosophila Ncd microtubule motor visualized in live embryos using Ncd-GFP fusion proteins. J. Cell Sci. 109:2429-2442.

22.Goto, S. and S. Hayashi. 1997. Cell migra- 
tion within the embryonic limb primordium of Drosophila as revealed by a novel fluorescence method to visualize mRNA and protein. Dev. Genes Evol. 207:194-198.

23.Griffin, B.A., S.R. Adams and R.Y. Tsien. 1998. Specific covalent labeling of recombinant protein molecules inside live cells. Science 281:269-272.

24.Haugland, R.P. 1996. Handbook of Fluorescent Probes and Research Chemicals, 6th ed. Molecular Probes, Eugene, Oregon.

25.Heim, R., D.C. Prasher and R.Y. Tsien. 1994. Wavelength mutations and posttranslational autoxidation of green fluorescent protein. Proc. Natl. Acad. Sci. USA 91:1250112504.

26.Hoyt, C. 1996. Liquid crystal filters clear the way for imaging multiprobe fluorescence. Biophotonics Int. July/Aug. 49-51.

27.Huang, Z. and S. Kunes. 1996. Hedgehog, transmitted along retinal axons, triggers neurogenesis in the developing visual centers of the Drosophila brain. Cell 86:411-422.

28.Inoue, S. 1995. Foundations of confocal scanned imaging in light microscopy, p. 1-17. In J.B. Pawley (Ed.), Handbook of Biological Confocal Microscopy, 2nd ed. Plenum Press, New York.

29.Inoue, S. and K.S. Spring. 1997. Video Microscopy: The Fundamentals, 2nd ed. Plenum Press, New York.

30.Kawasaki, E., M. Schermer and R. Zeleny. 1999. Confocal laser scanners for gene analysis. Biophotonics Int. March/April 47-49.

31.Kiehart, D.P., R.A. Montague, W.L. Rickoll, D. Foard and G.H. Thomas. 1994. Highresolution microscopic methods for the analysis of cellular movements in Drosophila embryos. Meth. Cell Biol. 44:507-532.

32.Knecht, D.A. 1993. Preparation of figures for publication using a digital color printer. BioTechniques 14:1006-1008.

33.Lipp, P. and E. Niggli. 1993. Ratiometric confocal calcium measurements with visible wavelength indicators in isolated cardiac myocytes. Cell Calcium 14:359-372.

34.Matsumoto, B. 1993. Cell biological applications of confocal microscopy. Meth. Cell Biol. 38:1-375.

35.Minsky, M. 1988. Memoir on inventing the confocal scanning microscope. Scanning 10:128-138.

36.Mohler, W.A., J.S. Simske, E.M. WilliamsMasson, J.D. Hardin and J.G. White. 1998. Dynamics and ultrastructure of developmental cell fusions in Caenorhabditis elegans hypodermis. Current Biology 8:1087-1090.

37.Mohler, W.A. and J.G. White. 1998. Stereo4-D reconstruction and animation from living fluorescent specimens. BioTechniques 24: 1006-1012.

38.Nellen, D., R. Burke, G. Struhl and K. Basler. 1996. Direct and long-range action of a DPP morphogen gradient. Cell 85:357-368.

39.Newman, E.A. and K.R. Zahs. 1997. Calcium waves in retinal glial cells. Science 275:844-847.

40.Niswander, K.D., S.M. Blackman, L. Rohde, M.A. Magnuson and D.W. Piston. 1995. Quantitative imaging of green fluorescent protein in cultured cells - comparison of microscopic techniques, use in fusion proteins and detection limits. J. Microsc. 180:109-116.

41.Paddock, S.W., S. Mahoney, M. Minshall, L. Smith, M. Duvic and D. Lewis. 1991. Improved detection of in situ hybridization by laser scanning confocal microscopy. BioTechniques 11:330-336.

42.Paddock, S.W., J.A. Langeland, P.J. DeVries and S.B. Carroll. 1993. Three-color immunofluorescence imaging of Drosophila embryos by laser scanning confocal microscopy. BioTechniques 14:42-48.

43.Paddock, S.W. 1994. Image manipulation. Confocal images to go? Curr. Biol. 4:857-860.

44.Paddock, S.W., P. DeVries, E. Buth and S.B. Carroll. 1994. Morphing: A new graphics tool for animating confocal images. BioTechniques 16:448-452.

45.Paddock, S.W., E.J. Hazen and P.J. DeVries. 1997. Methods and applications of three-color confocal imaging. BioTechniques 22:120-126.

46.Paddock, S.W. (Ed). 1998. Confocal Microscopy: Methods and Protocols. Methods in Mol. Biol. 122: Humana Press, Totowa, New Jersey.

47.Pawley, J.B. 1995. Handbook of Biological Confocal Microscopy, 2nd ed. Plenum Press, New York.

48.Pepperkok, R., A. Squire, S. Geley and P.I.H. Bastiaens. 1999. Simultaneous detection of multiple green fluorescent proteins in live cells by fluorescence lifetime imaging microscopy. Current Biology 9:269-272.

49.Piston, D.W., R.G. Summers, S.M. Knobel and J.B. Morrill. 1998. Characterization of involution during sea urchin gastrulation using two-photon excited photorelease and confocal microscopy. Microsc. Microanal. 4:404-414

50.Potter, S.M. 1996. Vital imaging: two photons are better than one. Current Biology 6:1595-1598.

51.Rizzuto, R., M. Brini, F. De Giorgi, R. Rossi, R. Heim, R.Y. Tsien and T. Pozzan. 1996. Double labeling of subcellular structures with organelle-targeted GFP mutants in vivo. Current Biology 6:183-188.

52.Sandison, D.R., R.M. Williams, K.S. Wells, J. Strickler and W.W. Webb. 1995. Quantitative fluorescence confocal laser scanning microscopy (CLSM), p. 39-53. In J.B. Pawley (Ed.), Handbook of Biological Confocal Microscopy, 2nd ed. Plenum Press, New York

53.Saunders, C. and R.S. Cohen. 1999. Double FISH and FISH- fluorescence immunolocalization procedures for whole-mount Drosophila ovaries. BioTechniques 26:186188 .

54.Scherson, T., G. Serbedzija, S. Fraser and M. Bronner-Fraser. 1993. Regulative capacity of the cranial neural tube to form neural crest. Development 118:1049-1061.

55.Schrock, E.S., T. du Manoir, B. Veldman, J. Schoell, M.A. Wienberg, Y. FergusonSmith, D.H. Ning, I. Ledbetter et al. 1996. Multicolor spectral karyotyping of human chromosomes. Science 273:494-497.

56.Sheppard, C.J.R. and D.M. Shotten. 1997. Confocal laser scanning microscopy. Royal Microscopical Society Handbook Series 38, Bios Scientific publishers, Oxford, UK.

57.Speicher, M.R., S.G. Ballard and D. Ward. 1996. Karyotyping human chromosomes by combinatorial multi-fluor FISH. Nature Genetics 12:368-375

58.Sullivan, K.F. and S.A. Kay. 1998. Green Fluorescent Proteins. Methods In Cell Biology, Vol. 58

59.Terasaki, M. and M.E. Dailey. 1995. Confocal microscopy of living cells, p. 327. In J.W. Pawley (Ed.), Handbook of Confocal Microscopy. Plenum Press, New York.

60.Thomas, C., P. DeVries, J. Hardin and J. White. 1996. Four-dimensional imaging: computer visualization of 3D movements in living specimens. Science 273:603-607.

61.Tsien, R.Y. 1989 Fluorescent indicators of ion concentrations. Meth. Cell Biol. 30:127-153.

62.Wanner, I., S.L. Baader, M. Brich, J. Oberdick and K. Schilling. 1997. Subcellular localization of specific mRNAs and their protein products in Purkinje cells by combined fluorescence, in situ hybridization and immunocytochemistry. Histochem. Cell Biol. 108:345-357.

63.Waterman-Storer, C.M., S.L. Shaw and E.D. Salmon. 1997. Production and presentation of digital movies. Trends In Cell Biol. 7:503-506.

64.White, J.G. and W.B. Amos. 1987. Confocal microscopy comes of age. Nature 328:183184.

65.White, J.G., W.B. Amos and M. Fordham. 1987. An evaluation of confocal versus conventional imaging of biological structures by fluorescence light microscopy. J. Cell Biol. 105:41-48.

66.White, J.G., V. Centonze, D. Wokosin and W. Mohler. 1997 Using multiphoton microscopy for the study of embryogenesis. Microscopy and Analysis 3:307-308

67.Wilson, T. 1990. Confocal Microscopy. Academic Press, San Diego, CA.

68.Wokosin, D.L., V.E. Centonze, S. Crittenden and J.G. White. 1995 Three-photon excitation of blue-emitting fluorophores by laser scanning microscopy. Molec. Biol. Cell 6:113a.

69.Xu, C., W. Zipfel, J.B. Shear, R.M. Williams and W.W. Webb. 1996. Multiphoton fluorescence excitation: new spectral windows for biological nonlinear microscopy. Proc. Nat. Acad. Sci. USA 93:10763-10768.

Address correspondence to:

Dr. Stephen W. Paddock

Department of Molecular Biology

University of Wisconsin

1525 Linden Drive

Madison, WI 53706, USA

Internet: paddock@facstaff.wisc.edu 